\title{
A Study of Inheritance in Progressive Intrahepatic Cholestasis: Hepatic excretory Function in Unaffected Family Members
}

\author{
PASCUAL BIDOT-LÓPEZ, DOUGLAS R. LABRECQUE, Y. EDWARD HSIA, \\ AND CAROLINE A. RIELY \\ Departments of Human Genetics, Pediatrics, and Internal Medicine, Yale University School of Medicine, New Haven, \\ Connecticut (USA)
}

\section{Summary}

In an attempt to identify the heterozygotes state for progressive intrahepatic cholestasis (PIC), hepatic excretory function $\left({ }^{131} \mathbf{I}\right.$ rose bengal half-life $(t / 2)$ and bromosulpthalein-transport maximum (BSP-Tm) was studied in controls and in eight members of a family, two of whom are affected with PIC. Values for ${ }^{131} I$ rose bengal $t / 2$ varied over a wide range in normal controls and were normal in patients with the syndrome of cholestasis and peripheral pulmonic stenosis in whom BSP-Tm and $45 \mathrm{~min} \%$ retention were abnormal. ${ }^{131} I$ rose bengal $t / 1 / 2$ was abnormal in seven of eight family members. Despite this, BSP studies, including Tm, percent retention at $\mathbf{4 5} \mathrm{min}$, clearance were normal in all unaffected family members with the exception of the mother who has a reduced BSP-Tm. Fasting serum bile acid studies were normal in all unaffected family members. These studies do not clearly define the inheritance in this syndrome and suggest that any of the following three possibilities exist: 1) that the methods employed were not sensitive enough to detect heterozygotes, 2) that the inheritance in this syndrome is heterogenous; for instance, compound heterozygotes or autosomal dominant, or 3 ) that the family studies here represents a syndrome different from PIC.

\section{Speculation}

It has been suggested that the mode of inheritance of progressive intrahepatic cholestasis is autosomal recessive. The use of sensitive tests of hepatic excretory function (such as the $t \frac{1}{2}{ }^{131}$ I rose bengal and the BSP-Tm may identify heterozygotes and confirm the type of inheritance.

The general heading of familial intrahepatic cholestasis includes a variety of syndromes such as cholestasis with peripheral pulmonic stenosis (also called arteriohepatic dysplasia) (1, 18), progressive intrahepatic cholestasis (the Byler Disease) $(3,4)$, a newly recognized syndrome associated with a presumed error in bile acid metabolism (7), and other, less well defined syndromes $(8,16)$.

PIC is characterized by recurrent attacks of jaundice, early onset of loose, foul-smelling stools, hepatosplenomegaly, growth failure, and, in some patients, mental retardation. Previous studies presented evidence for an autosomal recessive mode of inheritance (4).

In an attempt to distinguish heterozygotes, the authors have studied hepatic excretory function in the parents and four second degree relatives of two brothers with PIC. Investigations included percent retention, at $45 \mathrm{~min}$, clearance, and BSP-Tm ${ }^{131} \mathrm{I}$ rose bengal $t \frac{1}{2}$, and serum bile acid. The results are compared with those found in a control group including normal subjects and patients with other diseases affecting hepatic excretory function.

\section{MATERIALS AND METHODS}

\section{SUBJECTS}

Control. 1) Normal controls: In order to define normal values for ${ }^{131} I$ rose bengal $t^{1} / 2$, five normal controls were studied. These consisted of one child, a 2-yr-old female, and four young healthy adults, one female and three males, ages 30-33 yr. All had normal physical examinations and no history of liver disease. 2) Patients with diseases affecting hepatic excretory function: a) Biliary Atresia: E. W., an 11-wk-old male, and N. B., a 7-wk-old female, had total extrahepatic atresia documented at laparotomy. b) Cholestasis with peripheral pulmonary stenosis: R. D., Sr., and R. D., Jr., are a 27 -yr-old father and his infant son previously reported (12). J. C. is a 20 -yr-old female also affected with this cholestatic syndrome.

Family members with Progressive Intrahepatic Cholestasis: The parents, one maternal aunt and uncle, and the paternal aunt and grandmother of two brothers (J. O. and D. O.) previously reported (3) to have intrahepatic cholestasis were studied (Fig. 1). All had normal physical examinations with no previous history of liver disease. All the females studied were mothers with no history of pruritis during pregnancy. As part of the study, complete blood count, liver function tests, serum bile acids and lipids were obtained in all family members after $12 \mathrm{hr}$ of fasting. A chest $\mathrm{x}$-ray and an EKG were obtained in the parents of the two affected boys. Using 37 blood group factors, paternity could not be excluded.

\section{METHODS}

The ${ }^{131} I$ rose bengal $t \frac{1}{2}$ and BSP tests were done at least 3 wk apart to avoid problems in competition between the two organic anion dyes. Written consent for all investigations was obtained from all subjects under a protocol approved by the Human Investigation Committee.

$$
{ }^{131} \text { I ROSE BENGAL T1/2 }
$$

All subjects were given potassium iodide orally on the day before and the day of the iv ${ }^{131} I$ rose bengal $(10 \mu \mathrm{Ci})$. Blood samples were obtained immediately before the injection, then 24 , 48 , and $72 \mathrm{hr}$ after the injection. On the day of the injection, a cathartic was given to minimize irradiation to the colon. The radioactivity in the serum was counted in a gamma counter, and a semilog arithmetic plot of the results was constructed from which the serum half-life of ${ }^{131}$ I rose bengal was calculated.

\section{BSP STUDIES}

The BSP infusions were performed using the method described by Wheeler et al. (19). Subjects fasted for at least $12 \mathrm{hr}$ before the 


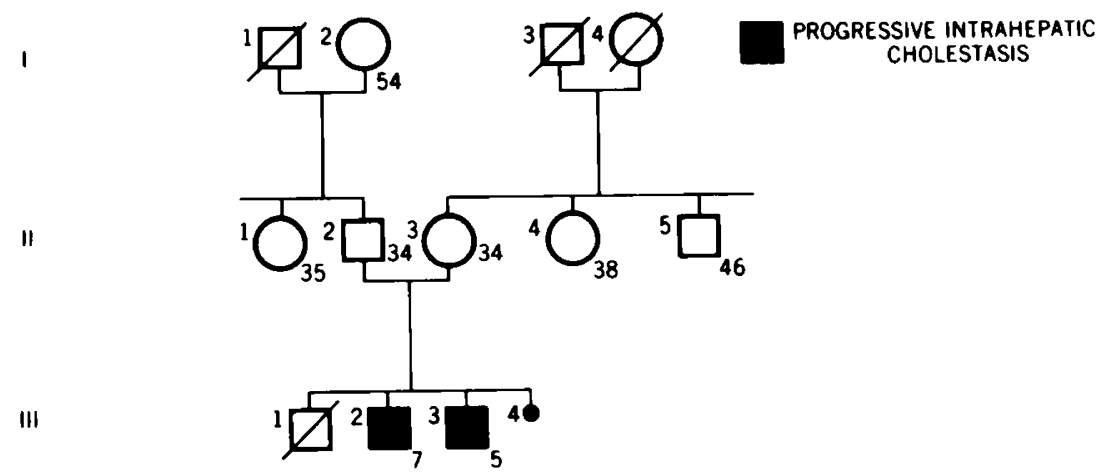

Fig. 1. Pedigree of family with PIC. The circle represents females, the small black circle indicates abortion, and the square represents males. The lower figures represent the age of that family member. Persons affected by the train are indicated by blacking in the symbols. All alive (without the slash) and unaffected members in this pedigree were studied.

study and remained supine during the $4 \mathrm{hr}$ of the study. First, a bolus of BSP $(5 \mathrm{mg} / \mathrm{kg}$ ideal body $\mathrm{wt})$ was given iv, and blood samples were obtained at $0,3,6,9,12,15,30,45$, and $60 \mathrm{~min}$ from the opposite arm for determination of plasma volume, percent of BSP retained, and BSP clearance $(5,19)$. Then, a constant infusion of BSP dissolved in $5 \%$ dextrose water was given at three different rates $(0.3,0.1$, and $0.2 \mathrm{mg} / \mathrm{min} / \mathrm{kg})$ over $1 \mathrm{hr}$ each. Blood samples were obtained every $10 \mathrm{~min}$ during the last $30 \mathrm{~min}$ of each infusion and were handled carefully to prevent hemolysis. The samples were analyzed for BSP content using preinjection samples of serum for the blanks and the maximum transport of BSP was calculated.

\section{SERUM BILE ACIDS}

Serum bile acids were quantified by gas liquid chromatography on a $2 \%$ OV-120 column, with internal standardization using the method of Ali and Javitt (2) to extract hydrolyse and methylate the bile acids; and the method of Roovers et al. (13) to prepare acetate derivatives.

\section{RESULTS}

The results of the ${ }^{131} I$ rose bengal $t / 2$ and BSP-Tm, $\%$ retention $45 \mathrm{~min}$ and clearance are presented in Table 1 .

\section{CONTROLS}

The ${ }^{131} I$ rose bengal $t^{1 / 2}$ in normal controls varied from 20.6 $46.4 \mathrm{hr}$ (means $\pm 2 \mathrm{SD}$ ). In the three patients with chelestasis and pulmonic stenosis, the ${ }^{131} I$ rose bengal $t^{1 / 2}$ values were within the normal limits although the BSP-Tm and \% retention at $45 \mathrm{~min}$ were abnormal in the two patients tested. The ${ }^{131} I$ rose bengal $t^{1 / 2}$ was abnormally prolonged in the two patients with biliary atresia.

\section{FAMILY MEMBERS}

Standard liver function tests, serum bile acids, coagulation profile, and serum lipids were all within normal limits in all the members of the family except the propositi. The chest $x$-ray and EKG in the parents of the affected boys were also normal. The ${ }^{131}$ I rose bengal was abnormally prolonged in all family members except the paternal aunt. The ${ }^{131} I$ rose bengal for the propositi was in the same range as that of the rest of the family members, even though these two boys have markedly abnormal liver function tests and serum bile acid concentrations. Despite the abnormalities in rose bengal metabolism, BSP studies including $\mathrm{Tm}, \%$ retention at $45 \mathrm{~min}$, and clearance, were normal in all family members with the exception of the mother, whose BSP-Tm was abnormally decreased to $3.7 \mathrm{mg} / \mathrm{min}$. In neither the controls nor in the family members was there any correlation between the abnormalities in ${ }^{131} I$ rose bengal and BSP-Tm.

\section{DISCUSSION}

Familial intrahepatic cholestasis is comprised of several distinct entities, characterized by cholestasis of unknown etiology in most of the cases studied. In this study, the authors examined the relatives of two brothers affected with progressive intrahepatic cholestasis (the Byler disease). The purpose of the study was to use sensitive tests of hepatic excretory function to confirm the pattern of inheritance in this family, and to identify heterozygotes.

Unlike renal excretory function, hepatic excretory function is difficult to assess, primarily because bile cannot be easily collected. Present methods involve observing the disappearance from plasma of substances which are extracted by the liver from plasma and are then excreted into bile. The organic anion dyes, rose bengal and BSP, are easy to measure in the serum, and are metabolis in a fashion similar to the natural organic anions bilirubin and bile acids. Like these natural organic anions, their plasma clearance is enhanced by treatment with phenobarbitol (9).

${ }^{131}$ I rose bengal $t^{1 / 2}$ in blood was studied by Thaler (17) in one normal subject and six patients with a variety of cholestatic syndromes, some of whom showed improvement on phenobarbital therapy. Previous studies from this laboratory have shown a similar improvement in rose bengal $t^{1 / 2}$ during phenobarbital therapy in the two brothers whose family is described in this report (11). In order to define the normal range for ${ }^{131}$ I rose bengal $t / 2$, five normal controls were also studied. These data demonstrate a wide range of normal values, and indicate that this test lacks sensitivity as a measure of hepatic excretion function.

BSP infusion tests have been extensively utilized, and normal values for $\mathrm{Tm}$ have been established by several authors $(10,14$, $15,19)$. Patients with a variety of liver diseases have abnormal BSP retention or $\operatorname{Tm}(10,12,14,15)$. It was found that the BSPTm was abnormally decreased in two patients with the syndrome of cholestasis and pulmonic stenosis (arteriohepatic dysphasia). These two patients both had ${ }^{131} I$ rose bengal $t \frac{1 / 2}{2}$ alues within the normal range. This lack of correlation between these two tests provides further evidence that the ${ }^{131} I$ rose bengal $t \frac{1}{2}$ is a poor measure of hepatic excretory function.

In the Byler disease, Clayton et al. (4) reported that two fathers of affected children, presumed to be obligatory heterozygotes, had abnormally decreased BSP-Tm. In several mothers of affected patients, there was abnormally high BSP percent of retention at 45 min during the last trimester of pregnancy, which suggested that heterozygotes could be detected by this test.

Our patients are affected with a type of the Byler disease presumably also inherited as an autosomal recessive condition. Standard liver functions tests, including serum bile acids, percent of BSP retention at $45 \mathrm{~min}$, and clearance of BSP were all normal in the family members. Furthermore, using ${ }^{131} I$ rose bengal $t \frac{1}{2}$ and BSP-Tm, the authors were unable to demonstrate consistent abnormalities in this family and the previous report's findings could not be confirmed. In this study, only in the mother of the affected 
Table 1. Results

\begin{tabular}{|c|c|c|c|c|}
\hline \multicolumn{5}{|c|}{ Controls } \\
\hline Subjects & $\begin{array}{c}\left.{ }^{131} \mathrm{I} \text { rose bengal } \mathrm{t}^{1 / 2} / \mathrm{hr}\right) \\
\end{array}$ & $\begin{array}{c}\text { BSP-Tm } \\
(\mathrm{mg} / \mathrm{min})\end{array}$ & $\begin{array}{l}\text { BSP \% retention } \\
\text { at } 45 \mathrm{~min}\end{array}$ & $\begin{array}{c}\text { BSP clearance } \\
\mathrm{ml} / \mathrm{min} / \mathrm{kg}\end{array}$ \\
\hline \multicolumn{5}{|l|}{ Normal } \\
\hline CAR & 26.1 & & & \\
\hline PB & 31.1 & & & \\
\hline DL & 37.9 & & & \\
\hline CG & 43.8 & & & \\
\hline AA & 28.8 & & & \\
\hline Normal Range & $20.6-46.4$ & $4.8-12.4(8)$ & $6.4-11.6(16)$ & $<4(9)$ \\
\hline \multicolumn{5}{|c|}{ Patients with diseases affecting hepatic excretory function } \\
\hline $\mathbf{E W}^{\prime}$ & 142.4 & & & \\
\hline$N^{\prime}$ & 69.6 & & & \\
\hline $\mathrm{RDjr}^{2}$ & 44.0 & & & \\
\hline RDsr $^{2}$ & 30.0 & 0.94 & 31.3 & \\
\hline $\mathrm{JC}^{2}$ & 30.0 & 2.30 & 33.5 & \\
\hline
\end{tabular}

${ }^{1}$ Biliary atresia.

${ }^{2}$ Cholestasis with peripheral pulmonary stenosis.

Table 2. Family with progressive familial intrahepatic cholestasis ${ }^{1}$

\begin{tabular}{lcccc}
\hline \multicolumn{1}{c}{ Subjects } & $\begin{array}{c}{ }^{131} \text { I rose } \\
\text { bengal } \mathrm{t}^{1 / 2} \\
(\mathrm{hr})\end{array}$ & $\begin{array}{c}\text { BSP-Tm } \\
(\mathrm{mg} / \mathrm{min})\end{array}$ & $\begin{array}{c}\text { BSP } \% \\
\text { retention } \\
\text { at 45 min }\end{array}$ & $\begin{array}{c}\text { BSP } \\
\text { Clearance } \\
\mathrm{ml} / \mathrm{min} / \mathrm{kg}\end{array}$ \\
\hline $\begin{array}{l}\text { Propositi } \\
\text { J. O. }\end{array}$ & 83 & & & \\
D. O. & 58 & & & \\
Parents & & & & \\
$\quad$ Mother & 61.3 & 3.7 & 5.4 & 9.28 \\
$\quad$ Father & 123.8 & 9.39 & 4.8 & 5.45 \\
Paternal & & & & \\
$\quad$ Grandmother & 84.5 & 6.4 & 3.4 & 4.65 \\
$\quad$ Aunt & 43.6 & & 6.0 & \\
Maternal & & & & \\
Aunt & 72.2 & 7.8 & 11.8 & 4.0 \\
Uncle & 77.9 & 8.9 & 5.8 & 4.0 \\
\hline
\end{tabular}

'For description of subjects, see text. Normal range for BSP-Tm represent previously reported values $\pm 2 \mathrm{SD}$.

brothers was the BSP-Tm reduced to values similar to those found in the unaffected fathers in the study of Clayton et al. (4). The father had an abnormal ${ }^{131}$ I rose bengal $t \frac{1}{2}$, but a normal BSP$\mathrm{Tm}$. The ${ }^{131}$ I rose bengal $t \frac{1}{2}$ of the family members did not differ from that of the two affected brothers (J. O., D. O.).

These results could have one of three explanations (excluding nonpaternity). First, it is possible that although the inheritance pattern of this family's cholestasis is autosomal recessive, neither the ${ }^{131} I$ rose bengal $t^{1 / 2}$ nor the BSP-Tm was a sensitive enough loading test to consistently detect the heterozygous state.

Second, it is possible that there is heterogeniety in the transmission of this syndrome. For example, the affected boys may be compound heterozygotes for two genes, one parent having a gene expressed by low BSP-Tm and the other with a gene expressed by prolonged rose bengal $t \frac{1}{2}$. Alternatively, it is possible that this disorder is autosomal dominant with variable penetrance that is expressed by a low BSP-Tm in the mother. Also, our results are consistent with sex linked recessive. However, affected females have been reported from both the inbred Amish (4), and from families in which consanguinity is denied (6). Finally, it is possible that the patients whose family is reported here do not have typical progressive intrahepatic cholestasis, but rather a new mutation in the very complex pathway for the excretion of bile acids and organic acids leading to a phenocopy of the Byler disease.

The results in this family study do not clearly define the inheritance of this syndrome. Further studies using these methods and others, such as bile acid kinetic studies, extended to other affected families will be necessary to gain greater understanding of this heterogeneous group of disorders.

\section{REFERENCES AND NOTES}

I. Alagille, D., Odièvre, M., Gautier, M., and Domingues, J. P.: Hepatic ductular hypoplasia associated with characteristic facies, vertebral malformations, retarded physical, mental, and sexual development, and cardiac murmur. J. Pediatr. 86: 63 (1975).

2. Ali, S. S., and Javitt, N. B.: Quantitative estimation of bile salt in serum. Can. J. Biochem. 48: 1054 (1970)

3. Ballow, M., Margolis, C. Z., Schachtel, B., and Hsia, Y. E.: Progressive familial intrahepatic cholestasis. Pediatrics, 51: 998 (1973).

4. Clayton, R. J., Iber, F. L., Ruebner, B. H., and McKusick, V. A.: Byler's disease. Fatal familial intrahepatic cholestasis in an Amish kindred. Amer. J. Dis. Child., 117: 112 (1969).

5. Goodman, R. C., and Kingsley, J. R: Sulfobromophthalein clearance test. J.A.M.A. 153: 462 (1953)

6. Gray, O. P., and Saunders, R. A.: Familial intrahepatic cholestatic jaundice in infancy. Arch. Dis. Childhood, 41: 320 (1966).

7. Hanson, R. F., Isenberg, J. N., Klein, P. D., and Sharp, H. L.: The metabolism of $3 \alpha, 7 \alpha, 12 \alpha$-trihydroty $5 \beta$ cholectan-26-oil acid in two brothers with cholestasis due to intrahepatic bile duct anomalies. J. Clin. Invest., 56: 577 (1975).

8. Juberg, R. C., Holland-Moritz, R. M., Henley, K. S., and Gonzalez, C. F. Familial intrahepatic cholestasis with mental and growth retardation. Pediatrics, 38: 819 (1966)

9. Klaassen, C.: Effects of phenobarbitol on the plasma disappearance and biliary excretion of drugs in rats. J. Pharmacol. Exptl. Ther., 175: 289 (1970).

10. Preisig, R., William, R., Sweeting, J., and Bradley, S.: Changes in BSP transport and storage. Amer. J. Med., 40: 170 (1966).

11. Riely, C. A., Ghent, E., LaBrecque, D., Hsia, Y. E., Bloomer, J. E., Klaskin, G.: Genetic and therapeutic heterogeneity in familial intrahepatic cholestasis. Gastroenterology, 72: 926 (1977).

12. Riely, C. A., LaBrecque, D. R., Ghent, C., Horwich, A., Klatskin, G.: A father and son with cholestasis and peripheral pulmonic stenosis: a distinct form of intrahepatic cholestasis. J. Pediatr., 92: 406 (1978).

13. Roovers, J., Evrard, E., Vanderhaeghe, H.: An improved method for measuring human blood bile acids. Clin. Chem. Acta, 19: 449 (1968)

14. Schoenfield, L. G., Foullk, W. T., and Butt, H. R.: Studies of sulfobromophthalein sodium (BSP) metabolism in man. I. In normal subjects and in patients with hepatic disease. J. Clin. Invest. 43: 7 (1964).

15. Shani, M., Gilon, E., Ben-Ezzer, J., Sheba, C.: Sulfobromophthalein tolerance test in patients with Dubin Johnson syndrome and their relatives. Gastroen terology, 59: 842 (1970).

16. Smith, D. W., Opitz, J. M., and Inhorn, S. L.: A syndrome of multiple developmental defects including polycystic kidneys and intrahepatic diliary dysgenesis in two siblings. J. Pediatr., 67: 617 (1965)

17. Thaler, M. M.: Effect of phenobarbitol on hepatic transport and excretion of ${ }^{131}$ rose bengal in children with cholestasis. Pediat. Res., 6: 100 (1972).

18. Watson, G. H., and Miller, V.: Arteriohepatic dysplasia: familial pulmonary arterial stenosis with neonatal liver disease. Arch. Dis. Childhood, 117: 459 (1973).

19. Wheeler, H. O., Meltzer, J. I., and Bradley, S. E.: Biliary transport and hepatic storage of sulfobromophthalein sodium in the unanesthetized dog, in normal man, and in patients with hepatic disease. J. Clin. Invest., 39: 113 (1960). 
20. The authors are indebted to Dr. Joseph R. Bloomer. Dr. Cameron N. Ghent, Dr. Brendan C. O’Malley, Ms. Barbara Kelly and Ms. Barbara Sparks for assistance.

21. Dr. Bidot is presently Assistant Professor of Internal Medicine, University of South Florida College of Medicine, Tampa, Florida, and whose address should be used for correspondence.

22. Dr. La Brecque is Presently Assistant Professor of Medicine. University of lowa lowa City, lowa.

23. Dr. Hsia is Professor of Genetics and Pediatrics, University of Hawaii.

Copyright $(\mathcal{1} 1979$ International Pediatric Research Foundation, Inc. 0031-3998/79/1309-1002 \$02.00/0
24. Dr. Riely is Assistant Professor of Internal Medicine and Pediatrics, Yale University School of Medicine.

25. This research was supported, in part, by the National Foundation Medical Services grant C-4I, USPHS grants AM 09527, and HD-00178-C7. Clinical studies were performed in the Yale Clinical Research Center, supported by grant RR-125, GCRC branch, Division of Research Resources, National Institutes of Health. Bethesda, MD (USA).

26. Received for publication March 2, 1978.

27. Accepted for publication August 30, 1978.

Printed in U.S.A. 